\title{
Chronic amiodarone administration causes liver damage via adipose tissue ER-stress dependent lipolysis, leading to hepatotoxic free fatty acid accumulation
}

\author{
Einav Hubel ${ }^{1}$, Sigal Fishman ${ }^{1}$, Minna Holopainen ${ }^{2}$, Reijo Kakela ${ }^{2}$, Ortal Shaffer ${ }^{1}$, Inbal \\ Houri $^{1}$, Roy Avraham ${ }^{1}$, Isabel Zvibel ${ }^{1}$, and Oren Shibolet ${ }^{1}$ \\ ${ }^{1}$ Tel Aviv Sourasky Medical Center \\ ${ }^{2}$ University of Helsinki
}

October 2, 2020

\begin{abstract}
Background and Purpose: Drug induced liver injury is an emerging form of acute and chronic liver disease that may manifest as fatty liver. Amiodarone (AMD), a widely used anti-arrhythmic drug, can cause hepatic injury and steatosis by a variety of mechanisms, which are not completely understood. Experimental Approach: Chronic damage was induced by daily gavage of $180 \mathrm{mg} / \mathrm{kg}$ body weight AMD for four consecutive days to C57Bl/6OlaHsd male mice. Key Results: Chronic AMD administration induced endoplasmic reticulum (ER) stress in both liver and adipose tissue. In adipose tissue, AMD reduced lipogenesis and increased lipolysis. Moreover, AMD treatment induced ER stress and ER stress-dependent lipolysis in 3T3L1 adipocytes in vitro. In the liver, AMD caused increased expression of genes encoding proteins involved in fatty acid (FA) uptake and transfer (Cd36, Fabp1 and Fabp4) and resulted in increased hepatic accumulation of free FAs, but not of triacylglycerols. In line with this, there was increased expression of hepatic de novo FA synthesis genes (Srebp1 and FA synthase encoded by Fasn). However, AMD significantly reduced the expression of the desaturase Scd1 and elongase Elovl6, detected at mRNA and protein levels. Accordingly, the FA profile of hepatic total lipids revealed increased accumulation of palmitate, a SCD1 and ELOVL6 substrate, and reduced levels of palmitoleate and cis-vaccenate, products of the enzymes. In addition, AMD-treated mice displayed increased hepatic apoptosis, known to be induced by lipotoxic palmitate. Conclusions and Implications: Chronic AMD induced ER stress and aggravated lipolysis in adipose tissue, while inducing a lipotoxic hepatic lipid environment.
\end{abstract}

\section{Hosted file}

Art 683009 20.pdf available at https://authorea.com/users/363870/articles/484528-chronicamiodarone-administration-causes-liver-damage-via-adipose-tissue-er-stress-dependentlipolysis-leading-to-hepatotoxic-free-fatty-acid-accumulation 

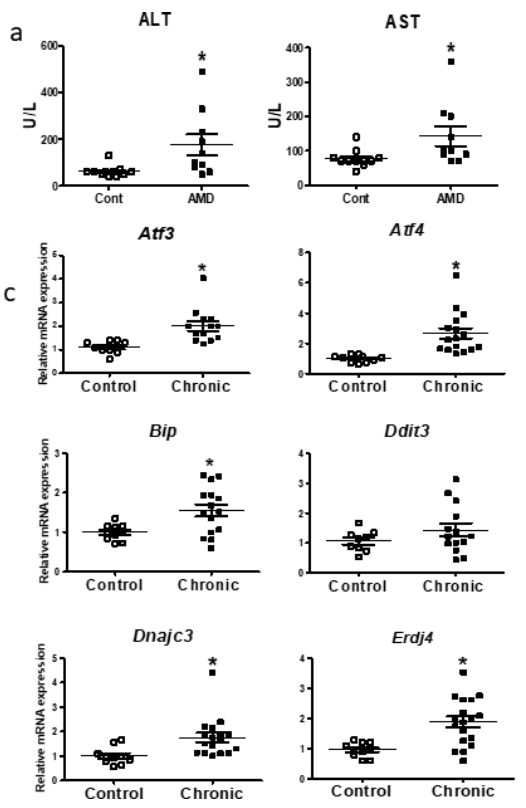

a
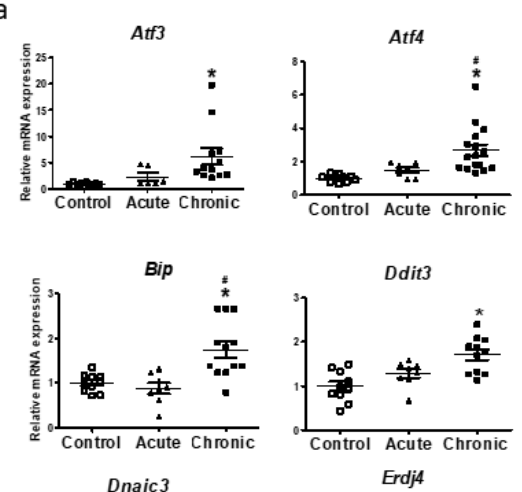

Dnajc3

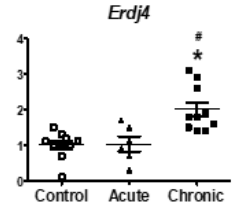

b

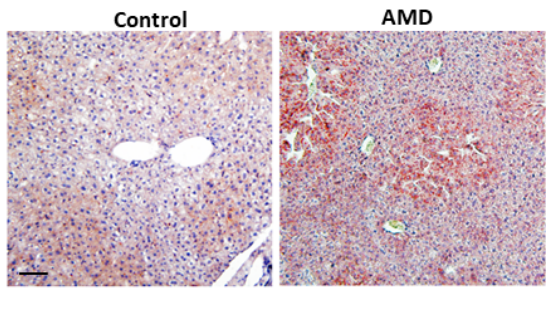

d

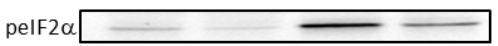

pIRE

p97
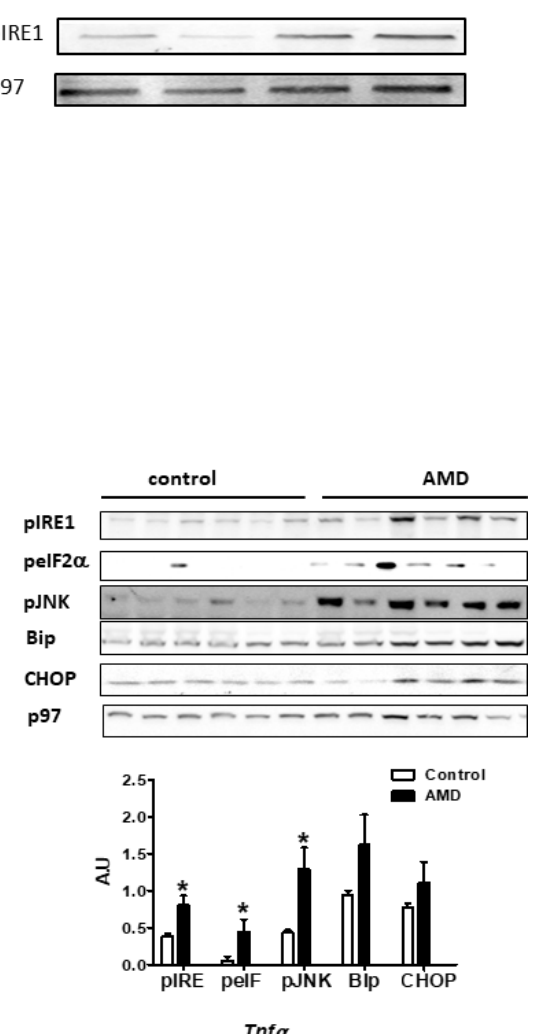

C

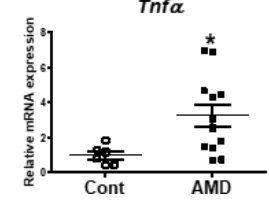



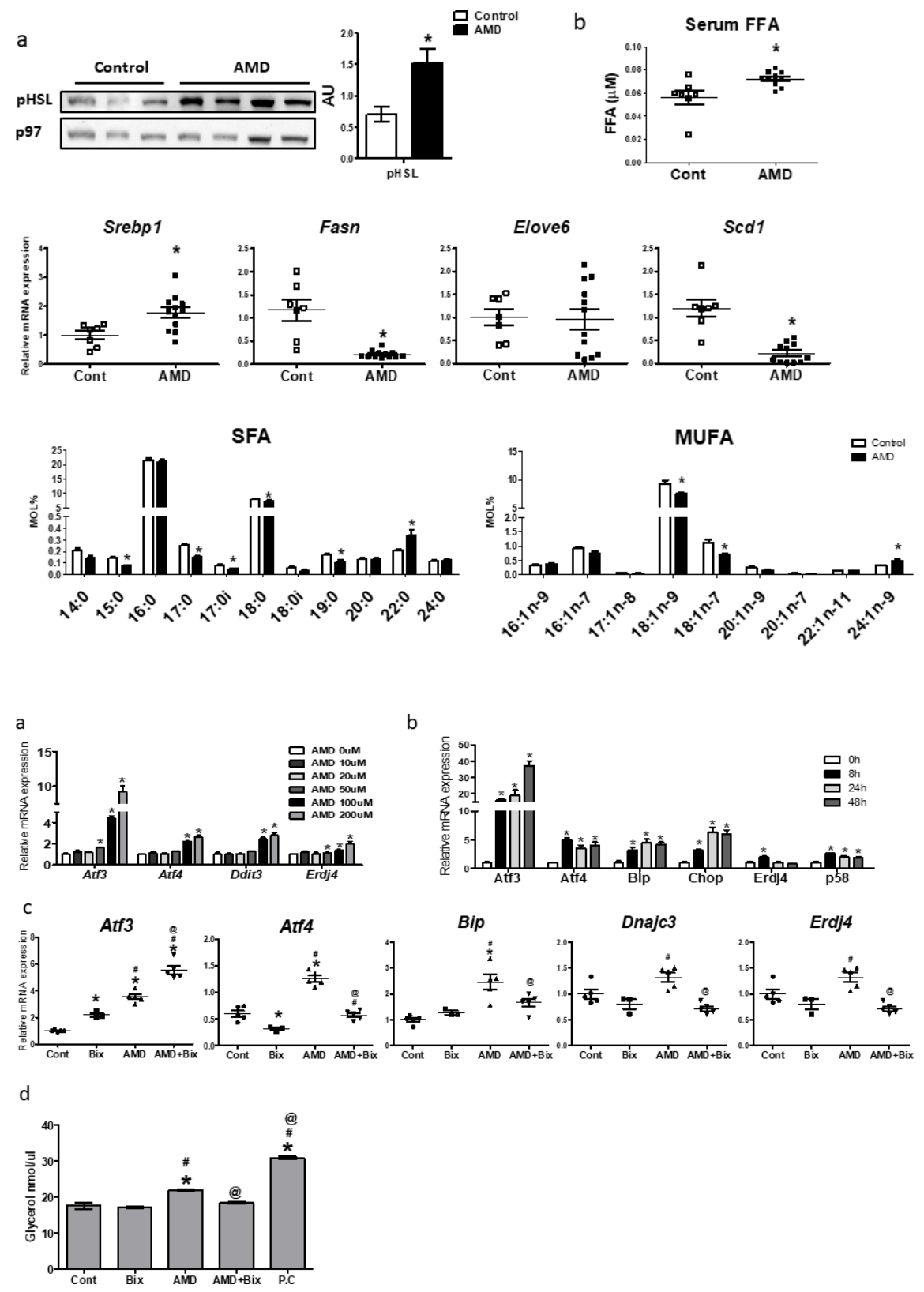

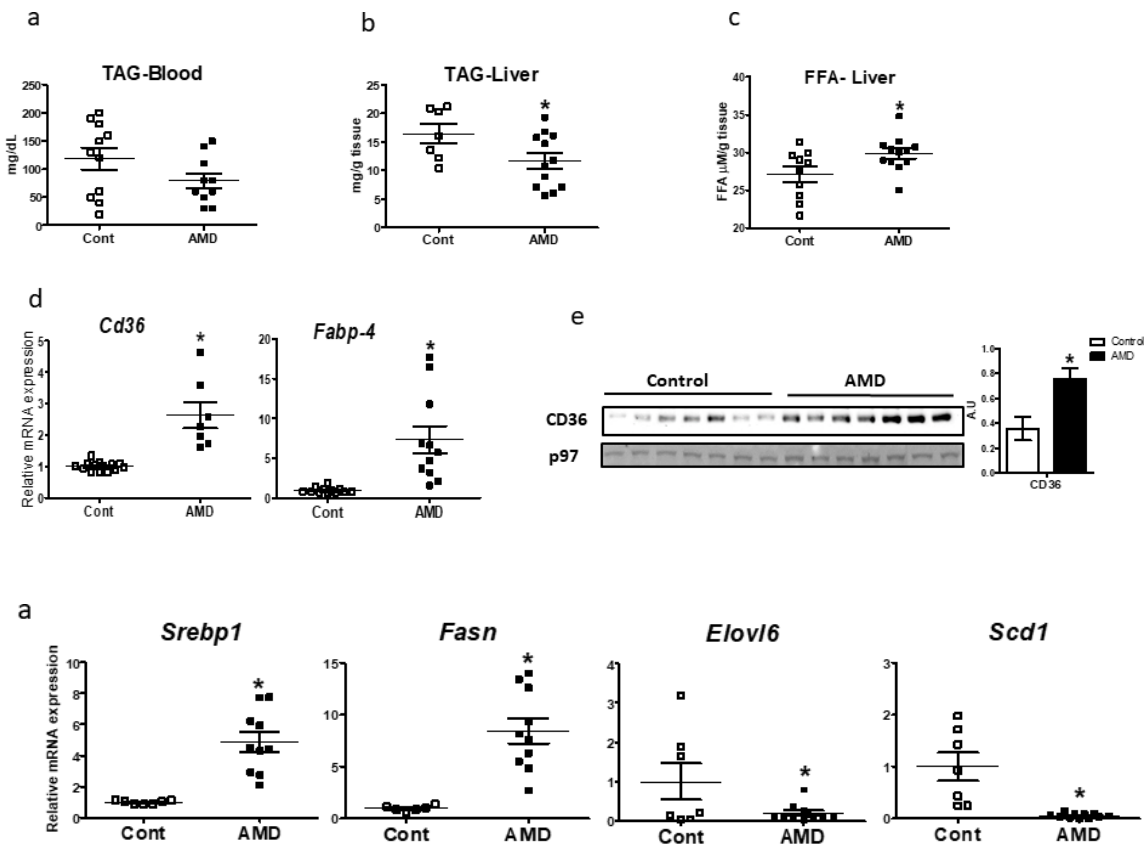

b

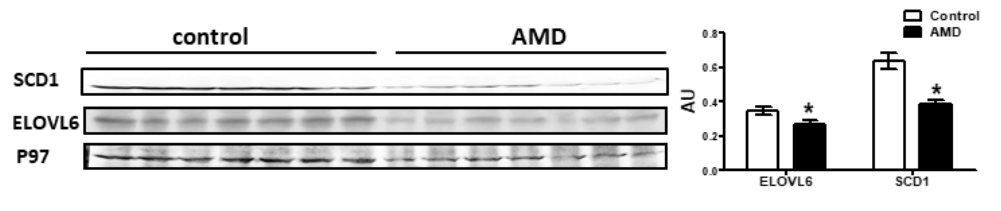

C

SFA
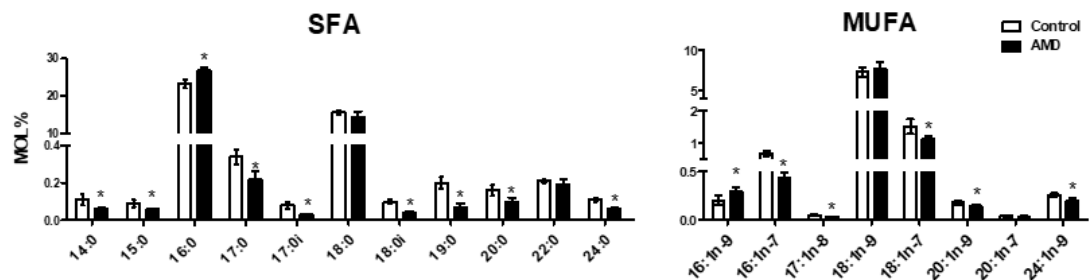

d

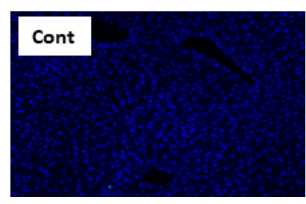

AMD 